\title{
Many-Particle Dephasing after a Quench
}

\author{
Thomas Kiendl ${ }^{1,2}$ and Florian Marquardt ${ }^{2,3}$ \\ ${ }^{1}$ Dahlem Center for Complex Quantum Systems and Institut für Theoretische Physik, Freie Universität Berlin, 14195, Berlin, Germany \\ ${ }^{2}$ Institute for Theoretical Physics, Universität Erlangen-Nürnberg, Staudtstraße 7, 91058 Erlangen, Germany \\ ${ }^{3}$ Max Planck Institute for the Science of Light, Günther-Scharowsky-Straße 1/Bau 24, D-91058 Erlangen, Germany
}

(Received 13 April 2016; revised manuscript received 2 February 2017; published 30 March 2017)

\begin{abstract}
After a quench in a quantum many-body system, expectation values tend to relax towards long-time averages. However, temporal fluctuations remain in the long-time limit, and it is crucial to study the suppression of these fluctuations with increasing system size. The particularly important case of nonintegrable models has been addressed so far only by numerics and conjectures based on analytical bounds. In this work, we are able to derive analytical predictions for the temporal fluctuations in a nonintegrable model (the transverse Ising chain with extra terms). Our results are based on identifying a dynamical regime of "many-particle dephasing," where quasiparticles do not yet relax but fluctuations are nonetheless suppressed exponentially by weak integrability breaking.
\end{abstract}

DOI: 10.1103/PhysRevLett.118.130601

Introduction.-The relaxation dynamics of quantum many-body systems has come under renewed scrutiny in the past years, due to its relevance for the foundations of thermodynamics and the availability of isolated systems, like cold atoms. The simplest case considers the evolution after a sudden quench of parameters [1]. Typically, one then analyzes local physical observables (like particle density, magnetization, currents), and asks about the time evolution of expectation values. The most basic question concerns the long-time averages after the quench: are they correctly described by a thermal state at some effective temperature related to the initial energy after the quench [2-17]? On the next, more refined level of analysis, we can study the timedependent fluctuations of expectation values around their temporal average. For any finite system, these persist even at infinite time. In principle, these represent a kind of longterm memory, since they are reproducible (the same for each repetition of the quench) and depend both on the exact time of the quench and on details of the initial state.

A crucial question for the foundations of statistical physics is, how are these fluctuations suppressed as a function of system size $N$, when approaching the thermodynamic limit? This is also relevant for experiments in equilibration, like analog quantum simulations carried out in finite ("mesoscopic") lattices.

These fluctuations around the time average are commonly characterized by $\sigma_{A}^{2}=\overline{\left[\langle\hat{A}(t)\rangle-\langle\hat{A}\rangle_{\mathrm{eq}}\right]^{2}}[5,18-20]$. The overbar denotes a time average and $\left\langle A \hat{\rangle}_{\mathrm{eq}}=\overline{\langle\hat{A}(t)\rangle}\right.$. Note that this is different from the quantum fluctuations $\operatorname{Var} \hat{A}(t)=\left\langle[\hat{A}(t)-\langle\hat{A}(t)\rangle]^{2}\right\rangle$, which are usually much larger and would be present even in a perfect thermal equilibrium state (where $\sigma_{A}^{2}$ vanishes).

The finite-size scaling of persistent temporal fluctuations after a quench has been approached so far from several angles: (i) in the context of the eigenstate thermalization hypothesis, justifying the neglect of off-diagonal contributions to expectation values [21-24]; (ii) based on the former, general mathematical bounds supplemented by physical arguments for generic interacting, nonintegrable systems $[18,19,25,26]$; (iii) calculations for simple integrable systems (which have, however, special properties that strongly differ from the generic case) [20,27-30]; (iv) numerics [31].

Here, we will provide exact analytical results for the suppression of fluctuations in a nonintegrable system, confirming the hypothesized exponential decay with system size. Our analysis rests on identifying a general dynamical regime which we term "many-particle dephasing," relevant for weak integrability breaking. The advantage over having purely numerical results will be that we can provide a complete description of how the result depends on the quench, the initial state, and parameters. The advantage vs analytical bounds is that the bounds are not guaranteed to be close to the true results.

Integrable transverse Ising model.-We start from the well-known integrable quantum Ising chain. We review, briefly, its properties and its quench dynamics, as they will be important for our analytical solution of the nonintegrable evolution later on. The quantum (transverse) Ising chain is an exactly solvable model for quantum phase transitions [32-37]:

$$
\hat{H}_{0}=\frac{\Omega}{2} \sum_{j=1}^{N} \hat{\sigma}_{z, j}-J \sum_{j=1}^{N} \hat{\sigma}_{x, j} \hat{\sigma}_{x, j+1} .
$$

Here $\hat{\sigma}_{x, j}$ and $\hat{\sigma}_{z, j}$ are spin-1/2 operators acting on site $j$. We will assume periodic boundary conditions, with $\hat{\sigma}_{x, N+1}=\hat{\sigma}_{x, 1}$. For $J<\Omega / 2$, the model is paramagnetic (where $\left\langle\hat{\sigma}_{z, j}\right\rangle<0$ ), while at $J=\Omega / 2$ there is a quantum phase transition into a ferromagnetic phase, with spins aligning either in the $+x$ or $-x$ direction. The model can be solved exactly by mapping to free fermions, via $\hat{\sigma}_{+, j}=\hat{c}_{j}^{\dagger} \exp \left(i \pi \sum_{l=1}^{j-1} \hat{c}_{l}^{\dagger} \hat{c}_{l}\right)$. This results in a quadratic 
fermionic Hamiltonian that does not conserve particle number and can be solved by Bogoliubov transformation in $k$ space:

$$
\hat{H}_{0}=\sum_{k}[\Omega-2 J \cos (k)] \hat{c}_{k}^{\dagger} \hat{c}_{k}-J i \sin (k)\left(\hat{c}_{k}^{\dagger} \hat{c}_{-k}^{\dagger}-\hat{c}_{-k} \hat{c}_{k}\right) .
$$

For definiteness we will assume $N$ is even. The quantization of wave numbers is slightly changed from the textbook case (due to an extra sign that enters when coupling site $N$ to site 1), with $k=2 \pi / N\left(l+\frac{1}{2}\right)$, where $l$ is an integer and $k$ ranges over the Brillouin zone $[-\pi, \pi[$. The Hamiltonian decomposes into independent sectors $(k,-k)$.

For this as well as other integrable models, it has been found that the temporal variance of many single-particle observables scales like $1 / N[20,28]$. However, there are important exceptions where there is no such suppression with $N$ [29]. In particular, one has to distinguish interacting systems mappable to noninteracting ones (the present case) and purely noninteracting systems. Only in the former, the complete one-particle density matrix relaxes [15].

In a quench of the coupling strength $J$ out of the prequench ground state, during the evolution we will have $(k,-k)$ either occupied by two particles or unoccupied. This can be viewed as an artificial spin $1 / 2$ system. We take $\hat{S}_{z k}=-1$ to correspond to $\left|0_{-k}, 0_{k}\right\rangle$ and $\hat{S}_{z k}=+1$ representing $\left|1_{-k}, 1_{k}\right\rangle=\hat{S}_{k}^{+}\left|0_{-k}, 0_{k}\right\rangle$, with $\hat{S}_{k}^{+} \equiv \hat{c}_{k}^{\dagger} \hat{c}_{-k}^{\dagger}$. In that notation, the Hamiltonian, Eq. (2), becomes a set of decoupled effective spin- $1 / 2$ systems:

$$
\hat{H}_{0}=\frac{1}{2} \sum_{k>0} \Omega_{k} \vec{b}_{k} \hat{\vec{S}}_{k}
$$

We introduced the two-particle excitation frequencies $\Omega_{k}=\sqrt{[2 \Omega-4 J \cos (k)]^{2}+[4 J \sin (k)]^{2}}$, and the field direction $\quad \vec{b}_{k}=[0,4 J \sin (k), 2 \Omega-4 J \cos (k)]^{T} / \Omega_{k}$. The ground and excited state, $\left| \pm_{k}\right\rangle$, have energies $\pm \Omega_{k} / 2$, and $\left\langle \pm_{k}\left|\hat{\vec{S}}_{k}\right| \pm_{k}\right\rangle= \pm \vec{b}_{k}$. In this picture, a quench corresponds to a sudden change of $\Omega_{k}$ and $\vec{b}_{k}$, such that for each $k$ the Bloch vector starts to precess around the new field direction: $d\left\langle\hat{\vec{S}}_{k}\right\rangle / d t=\Omega_{k} \vec{b}_{k} \times\left\langle\hat{\vec{S}}_{k}\right\rangle$.

An example for an observable is the projector for the spin pointing along $+z$ at some site $j: \hat{A}=\hat{\sigma}_{+, j} \hat{\sigma}_{-, j}$. Because of translational invariance, $\langle\hat{A}(t)\rangle$ is independent of $j$. One finds $\langle\hat{A}(t)\rangle=N^{-1} \sum_{k>0}\left(\left\langle\hat{S}_{z k}\right\rangle+1\right)$, resulting in an expression of the form $\langle\hat{A}(t)\rangle=N^{-1} \sum_{k>0}\left[A_{0 k}+\right.$ $\left.A_{c k} \cos \left(\Omega_{k} t\right)+A_{s k} \sin \left(\Omega_{k} t\right)\right]$. At sufficiently long times, all the oscillatory terms dephase, producing seemingly random time-dependent fluctuations. This process can be termed "single-particle dephasing," since it results from the superposition of different oscillation frequencies whose number scales linearly with system size.

Thus, the temporal variance ends up being $\sigma_{A}^{2}=N^{-2} \sum_{k>0}\left(A_{c k}^{2}+A_{s k}^{2}\right) / 2$. In the limit of large $N$, this becomes

$$
\sigma_{A}^{2}=N^{-1} \int_{0}^{\pi} \frac{d k}{2 \pi}\left(A_{c k}^{2}+A_{s k}^{2}\right) / 2,
$$

i.e., $\sigma_{A}^{2} \sim N^{-1}$, confirming the result of Ref. [20].

Quench in the nonintegrable model.-The general physical expectation for nonintegrable systems is that the long-time steady state after a quench has fluctuations that are exponentially suppressed in particle number (system size) $N$, in contrast to the power-law suppression in the integrable case displayed above. This was made explicit first in Ref. [18]. There, an upper bound was derived, $\sigma_{A}^{2} \leq\left(a_{\max }-a_{\min }\right)^{2}$. IPR. Here, $a_{\max }$ and $a_{\min }$ are the maximum and minimum eigenvalues of $\hat{A}$. IPR $=$ $\sum_{n}\left|\left\langle\Phi_{n} \mid \Psi(0)\right\rangle\right|^{4}$ denotes the inverse participation ratio, which decreases if the initial state $|\Psi(0)\rangle$ spreads over more energy eigenstates $\left|\Phi_{n}\right\rangle$. It was then argued on general physical grounds that the IPR usually decreases exponentially with system size. However, an argument of this kind does not reveal how fast the decay is for any concrete system or quench scenario, or whether the upper bound displays the correct parameter dependence at all, since it will not be tight in general. More recently, it was reported that numerical simulations for a variety of models and quench scenarios indeed reveal an exponential suppression with system size, for the finite-size systems that could be addressed [31].

Our goal here is to go beyond bounds and numerics, and to find an analytical expression for a nonintegrable case. We break the integrability of the quantum Ising model by adding next-nearest-neighbor (NNN) coupling $\hat{H}_{\mathrm{NNN}}=-J_{\mathrm{NNN}} \sum_{j} \hat{\sigma}_{x, j} \hat{\sigma}_{x, j+2}$. In the fermion representation, this gives rise to two-particle interactions. Other choices for integrability breaking are possible, which we will address later. A direct numerical simulation [Fig. 1] indeed reveals a stronger suppression of fluctuations, that seems to be consistent with an exponential decay in $N$.

We now come to an important question: What is the physical origin of this strong suppression? Initially, one might suspect "true thermalization," in the sense of inelastic scattering of quasiparticles leading to a redistribution of quasiparticle populations. This process could then be
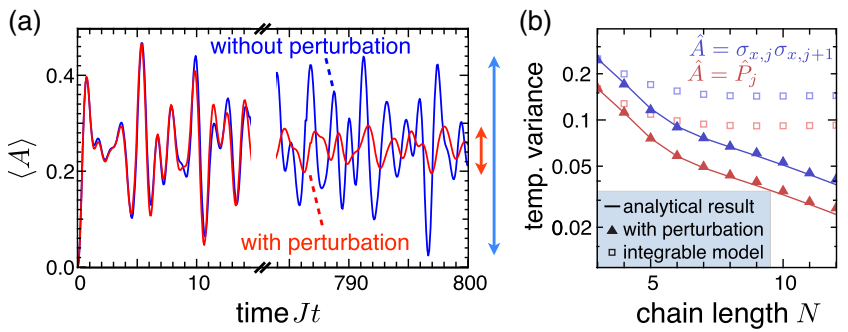

FIG. 1. Many-particle dephasing in a chain with $N=12$. The quench jumps from $J_{\text {pre }} / \Omega=0$ to $J / \Omega=0.8$. Without perturbation (blue) the fluctuations at early and late times are similar. A weak NNN coupling of strength $J_{\mathrm{NNN}} / J=0.01$ leads to a significant additional relaxation for $t \rightarrow \infty$. (b) The temporal variance $N \sigma_{A}^{2}$ for two different observables shows an exponential decay in $N$. [Analytical result from Eq. (6).] 
described using a kinetic equation, and the final state would be thermal. However, the simulation shows that this is not the case, the quasiparticle distribution remains practically unchanged. There is further numerical evidence that we are not witnessing thermalization: The fluctuations decay to their steady-state long-time limit during a time scale $\tau^{*}$ that scales linearly in the inverse perturbation: $\tau^{*} \sim\left|J_{\mathrm{NNN}}\right|^{-1}$. This is in contrast to the behaviour expected from a kinetic equation, where the relaxation rate would be set by $J_{\mathrm{NNN}}^{2}$.

Many-particle dephasing.-Instead, we have identified a mechanism that could be termed "many-particle dephasing." First, we note that, for weak interactions, the many-body energy eigenstates still coincide to a very good approximation with those of the integrable model. This explains the absence of thermalization in the occupations of quasiparticles. At the same time, however, the energies are changed. This lifts the exponentially large degeneracies of the integrable model and gives rise to dephasing. The number of frequencies involved is now exponentially large in $N$, which is the reason we term the resulting dynamics "manyparticle dephasing." The generic situation, including the different time scales, is shown schematically in Fig. 2.

An effect similar to the many-particle dephasing mechanism has been predicted for a chain of harmonic oscillators that are perturbed weakly by an anharmonic interaction [38]. In this bosonic model, the weak anharmonicity leads to an exponential number of available frequencies, causing an exponential decay of the fluctuation strength.

It can be shown easily (e.g., Ref. [18]) that fluctuations in the long-time limit obey $\sigma_{A}^{2}=\sum_{\Delta \neq 0}\left|\sum_{\Delta_{\alpha}=\Delta} A_{\alpha}\right|^{2}$. Here $\alpha=(f, i)$ denotes a transition between two energy eigenstates $i$ and $f$ where $\Delta_{\alpha}=E_{f}-E_{i}$ is the transition energy, and $A_{\alpha}=\Psi_{f}^{*} A_{f i} \Psi_{i}$ combines the transition matrix element of the observable with the amplitudes $\Psi_{l}=\left\langle\Phi_{l} \mid \Psi(0)\right\rangle$ of the initial state with respect to the postquench energy eigenbasis $\Phi_{l}$.

We now consider an arbitrary transition $i \rightarrow f$ that is induced by $\hat{A}$. Suppose the observable just affects a single quasiparticle at a time, or (in our case) it affects only a

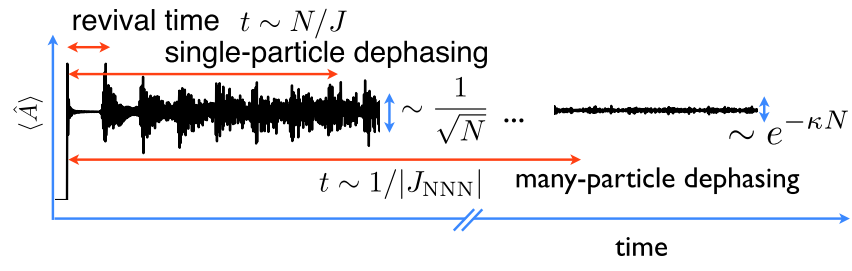

FIG. 2. Schematic overview of different dynamical regimes after a quench in a finite-size system that is weakly perturbed away from an integrable, effectively noninteracting model (displayed for a local observable in the perturbed transverse Ising model, but valid more generally). First, revivals occur. Second, a transient steady-state with fluctuations $\sigma_{A} \sim 1 / \sqrt{N}$ is observed, as predicted for the integrable case. Finally, after a time that scales as the inverse of the integrability-breaking perturbation, the final steady state is reached. There, fluctuations are reduced exponentially in the system size, due to many-particle dephasing. single $(k,-k)$ pair of states. All other quasiparticles (or $k$ pairs) are merely spectators. Such a structure is typical for single-particle observables. It is at this point that the weak integrability-breaking interactions impose a crucial difference. For the integrable (effectively noninteracting) case, there is an exponentially large number of other transitions that have the same transition energy. These are obtained by picking all possible configurations of the remaining "spectator" degrees of freedom (which are identical in the initial and final state). In contrast, for the nonintegrable (weakly interacting) case, there is a correction to the transition energies which lifts this massive degeneracy. For the present model, the transition energy correction $\delta \Delta_{f i} \sim J_{\mathrm{NNN}}$ turns out to be a sum over contributions that depend on pairs of occupation numbers, $n_{k}$ and $n_{k^{\prime}}$ (see Supplemental Material [39]). Given a change in one of the occupation numbers, the correction thus depends on the configuration of all the "spectator" degrees of freedom. Therefore, barring any (rare) accidental degeneracies, the initial degeneracy is completely lifted. That statement is confirmed by direct numerical inspection of $\delta \Delta_{f i}$.

Assuming that all the transition energies $\Delta_{\alpha}$ have become nondegenerate, we find $\sigma_{A}^{2}=\sum_{f \neq i}\left|\Psi_{f}^{*} A_{f i} \Psi_{i}\right|^{2}$. In general, it would still be an impossible task to evaluate this expression analytically. At this stage, however, the important observation is that the $\Delta_{\alpha}$ do not enter any more, even though their modification by the weak interaction was crucial to lift the degeneracies. Our strategy will be to evaluate this expression for the matrix elements calculated with respect to the unperturbed integrable many-particle eigenfunctions. In this way, we will arrive at analytical insights into the suppression of fluctuations for the nonintegrable model. The requirement for this to work is that the perturbation $J_{\mathrm{NNN}}$ is still weak, such that the eigenfunctions have not been changed appreciably. Later we will check the results against numerics. We note that a similar approach has been used before by Rutkevich for a chain of harmonic oscillators with weak anharmonic interactions [38].

Each energy eigenstate of the integrable transverse Ising model can be written as a product state: $\left|\Phi_{n}\right\rangle=\Pi_{k>0}|\varphi(n, k)\rangle$. Each configuration $n$ is described by $N / 2$ bits $\varphi(n, k) \in\{-1,+1\}$, where -1 denotes the ground state $\left|--_{k}\right\rangle$ and +1 the excited state $\left|+_{k}\right\rangle$ in the $(k,-k)$ sector. The observable we focused on in the numerical example was $\hat{A}=\left(\hat{\sigma}_{z, j=0}+1\right) / 2$, which, in fermionic language, is equal to $\hat{A}=N^{-1} \sum_{k, k^{\prime}} \hat{c}_{k}^{\dagger} \hat{c}_{k^{\prime}} \exp \left[-i\left(k-k^{\prime}\right) x\right]$. For this observable, we find

$$
\left\langle\Phi_{m}|\hat{A}| \Phi_{n}\right\rangle=\frac{2}{N} \sum_{k>0}\left\langle\varphi(m, k)\left|\hat{S}_{k}^{+} \hat{S}_{k}^{-}\right| \varphi(n, k)\right\rangle I_{k},
$$

where $I_{k} \equiv \Pi_{k^{\prime} \neq k} \delta_{\varphi\left(m, k^{\prime}\right), \varphi\left(n, k^{\prime}\right)}$ enforces the initial and final configurations of spectators $k^{\prime} \neq k$ to match.

In evaluating the general formula for $\sigma_{A}^{2}$, we have to sum over all possible many-particle transitions $i \rightarrow f$. However, the Kronecker delta in Eq. (5) enforces the configurations $\varphi\left(i, k^{\prime}\right)$ and $\varphi\left(f, k^{\prime}\right)$ to be equal except at $k^{\prime}=k$. We still 
have to sum over exponentially many configurations, though that can be handled by regrouping terms and a bit of combinatorics (see Supplemental Material [39]). In doing this, we exploit the fact that the initial state can be written as a product state over the different $k$ sectors (since it is an eigenstate of the prequench Hamiltonian).

Analytical results.-The final analytical result for the long-term, steady-state fluctuations in the weakly nonintegrable model ( small $J_{\mathrm{NNN}} \neq 0$ ) is

$$
\sigma_{A}^{2}=\frac{\mathcal{C}}{N} \exp (-2 \kappa N) .
$$

Here the exponential is equal to the IPR. We find explicitly $2 \kappa=-(1 / N) \sum_{k>0} \ln \operatorname{IPR}(k)$, where $\operatorname{IPR}(k)=\left|\left\langle+_{k} \mid \Psi_{k}\right\rangle\right|^{4}+$ $\left|\left\langle-{ }_{k} \mid \Psi_{k}\right\rangle\right|^{4}$ is the IPR for the initial state $\left|\Psi_{k}\right\rangle$ in sector $k$. In the limit of large $N, \kappa$ becomes $N$ independent: $2 \kappa \rightarrow \int_{0}^{\pi}(d k / 2 \pi) \ln \operatorname{IPR}(k)$, giving us analytical access to the exponential suppression of fluctuations.

The prefactor in $\sigma_{A}^{2}$ contains a further $1 / N$ suppression, and a constant $\mathcal{C}$, which can be given explicitly as well:

$$
\mathcal{C}=\frac{8}{N} \sum_{k>0} w(k) \approx 8 \int_{0}^{\pi} \frac{d k}{2 \pi} w(k),
$$

where

$$
w(k)=\operatorname{IPR}^{-1}(k)\left|\left\langle+_{k}\left|\hat{S}_{k}^{+} \hat{S}_{k}^{-}\right|-{ }_{k}\right\rangle\right|^{2} P_{k}\left(1-P_{k}\right),
$$

and $P_{k}=\left|\left\langle+_{k} \mid \Psi_{k}\right\rangle\right|^{2}$.

Writing the Ising Hamiltonian in the form of Eq. (3), we can give explicit expressions in terms of the "magnetic field" directions before $\left(\vec{b}_{k}^{\prime}\right)$ and after $\left(\vec{b}_{k}\right)$ the quench:

$$
\begin{gathered}
\operatorname{IPR}(k)=\frac{1}{2}\left[1+\left(\vec{b}_{k} \vec{b}_{k}^{\prime}\right)^{2}\right] \\
w(k)=\frac{\left(1-b_{z k}^{2}\right)\left[1-\left(\vec{b}_{k} \vec{b}_{k}^{\prime}\right)^{2}\right]}{16 \operatorname{IPR}(k)} .
\end{gathered}
$$

We find a very good agreement between these analytical expressions and numerical results for finite system sizes
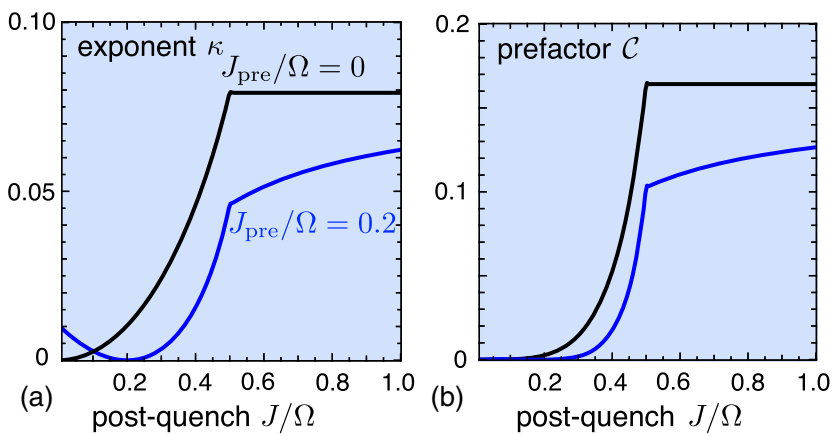

FIG. 3. Analytical predictions, depending on the postquench parameter $J$. (a) Decay constant $\kappa$ for the decay of fluctuations with system size, and (b) prefactor $\mathcal{C}$ (see main text, in the formal limit $N \rightarrow \infty$ ). The quench assumed here jumps from a coupling $J_{\text {pre }}$ to $J$.
(Fig. 4). The prefactor and the decay coefficient are shown in Fig. 3. Note that both behave nonanalytically at the quantum critical point.

For other observables, similar calculations can be done. For example, for $\sigma_{x, j} \sigma_{x, j+1}$, the result is the same up to the change $\hat{S}_{k}^{+} \hat{S}_{k}^{-} \mapsto 2 \cos (k) \hat{S}_{k}^{+} \hat{S}_{k}^{-}-\sin (k) \hat{S}_{y k}$ in Eq. (8).

Time scale.-The many-particle dephasing rate is expected to be proportional to the transition energy splitting, which in the above case is of order $J_{\mathrm{NNN}}$. Numerical results are compatible with this expectation. Moreover, they indicate that the corresponding time scale is approximately independent of $N$, as far as we could ascertain (an increase of the chain length by a factor of more than two leaves this time scale approximately constant; see Supplemental Material [39]). Once the system size becomes large enough such that quasiparticles can mix, we anticipate the onset of Boltzmann dynamics at a time scale $\sim J_{\mathrm{NNN}}^{-2}$, which will be much larger for weak integrability breaking.

Other variants and models.-We expect our approach to work for a very general class of integrability breaking terms: they only have to lift the single-particle degeneracy. The case of long-range coupling is discussed in the Supplemental Material [39] and fits well with our analytical prediction. Furthermore we considered a weak longitudinal field $\hat{V}_{x}=J_{x} \sum_{j=1}^{N} \sigma_{x, j}$. In the paramagnetic phase, this yields good agreement with our prediction, see Fig. 4. Exceptions occur when the inversion symmetry is spontaneously broken in the ferromagnetic phase (see the Supplemental Material [39] for a general discussion on the applicability in the case of spontaneous symmetry breaking).

Other examples include systems of free bosons or fermions in any dimension. Introducing a weak interaction will lift the initial massive degeneracy, leading to manyparticle dephasing as described here.
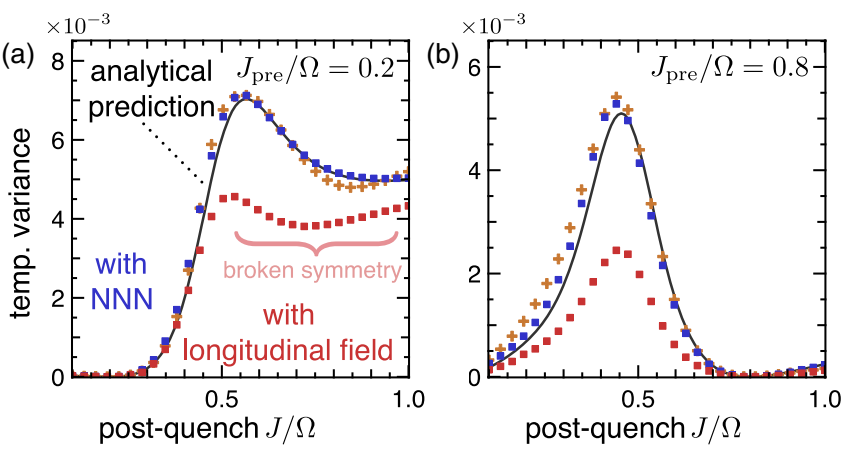

FIG. 4. Temporal variance $\sigma_{A}^{2}$ for $N=8$ and different prequench parameters. The squares show results from numerical exact diagonalization. Integrability is weakly broken by $J_{\mathrm{NNN}} / \Omega=$ 0.01 (blue) or a longitudinal magnetic field $J_{x} / \Omega=0.01$ (red). The black line shows the analytical predictions. With the pre- or postquench parameters in the ferromagnetic phase and nonzero $J_{x}$, stronger deviations due to the spontaneous symmetry breaking occur. For smaller fields, the agreement is restored (orange crosses) $\left[J_{x} / \Omega=2 \times 10^{-4}\right.$ in (a) and $J_{x} / \Omega=5 \times 10^{-4}$ in (b) $]$. 
Our theory may also provide insights into disordered systems. If they display Anderson localization in the integrable case and remain many-body localized after switching on the interaction, we conjecture that the evolution after a quench can also show signs of manyparticle dephasing. The system size would be replaced by the localization length, such that we are effectively dealing with many independent finite-size mesoscopic systems.

Experimental implementation.-Our predictions might be tested in both chains of trapped ions and superconducting qubit arrays. We have estimated that such tests are within the reach of feasible experimental parameters (see Supplemental Material [39], especially Fig. 4), and the long-range coupling in ions in particular offers a new variation on the scenario described here.

Conclusions. - Exploring time-dependent fluctuations represents the important next step beyond the discussion of long-time averages. We discovered both a new dynamical regime and an analytical way of predicting such fluctuations and their dependence on system size. The underlying mechanism should apply whenever one starts from an effectively free model, where the many-particle energies are highly degenerate, which are then split by weak integrability-breaking interactions.

We expect our results to encourage experimentalists, in particular ones using trapped ions or superconducting qubits, to address the outstanding experimental problem of systematically studying the decay of fluctuations and comparing them to both numerical and analytical predictions.

We thank Marcos Rigol, Lea Santos, and Aditi Mitra for discussions. T. K. acknowledges financial support from the Helmholtz Virtual Institute "New states of matter and their excitations."

[1] A. Polkovnikov, K. Sengupta, A. Silva, and M. Vengalattore, Rev. Mod. Phys. 83, 863 (2011).

[2] M. Rigol, V. Dunjko, V. Yurovsky, and M. Olshanii, Phys. Rev. Lett. 98, 050405 (2007).

[3] M. Rigol, V. Dunjko, and M. Olshanii, Nature (London) 452, 854 (2008).

[4] J. M. Deutsch, Phys. Rev. A 43, 2046 (1991).

[5] M. Srednicki, Phys. Rev. E 50, 888 (1994).

[6] H. Tasaki, Phys. Rev. Lett. 80, 1373 (1998).

[7] S. R. Manmana, S. Wessel, R. M. Noack, and A. Muramatsu, Phys. Rev. Lett. 98, 210405 (2007).

[8] M. Cramer, C. M. Dawson, J. Eisert, and T. J. Osborne, Phys. Rev. Lett. 100, 030602 (2008).

[9] C. Kollath, A. M. Läuchli, and E. Altman, Phys. Rev. Lett. 98, 180601 (2007).

[10] M. A. Cazalilla, Phys. Rev. Lett. 97, 156403 (2006).

[11] T. Barthel and U. Schollwöck, Phys. Rev. Lett. 100, 100601 (2008).

[12] C. Neuenhahn and F. Marquardt, Phys. Rev. E 85, 060101 (2012).

[13] L. F. Santos and M. Rigol, Phys. Rev. E 82, 031130 (2010).
[14] J. Eisert, M. Friesdorf, and C. Gogolin, Nat. Phys. 11, 124 (2015).

[15] T. M. Wright, M. Rigol, M. J. Davis, and K. V. Kheruntsyan, Phys. Rev. Lett. 113, 050601 (2014).

[16] M. Srednicki, arXiv:cond-mat/9410046.

[17] E. J. Torres-Herrera, D. Kollmar, and L. F. Santos, Phys. Scr. 2015, 014018 (2015).

[18] P. Reimann, Phys. Rev. Lett. 101, 190403 (2008).

[19] A. J. Short, New J. Phys. 13, 053009 (2011).

[20] L. C. Venuti and P. Zanardi, Phys. Rev. E 87, 012106 (2013).

[21] M. Srednicki, Phys. Rev. E 50, 888 (1994).

[22] M. Srednicki, J. Phys. A 32, 1163 (1999).

[23] M. Rigol, V. Dunjko, and M. Olshanii, Nature (London) 452, 854 (2008).

[24] M. Rigol, Phys. Rev. A 80, 053607 (2009).

[25] P. Reimann, Phys. Scr. 86, 058512 (2012).

[26] A. J. Short and T. C. Farrelly, New J. Phys. 14, 013063 (2012).

[27] L. Campos Venuti and P. Zanardi, Phys. Rev. E 89, 022101 (2014).

[28] A. C. Cassidy, C. W. Clark, and M. Rigol, Phys. Rev. Lett. 106, 140405 (2011).

[29] C. Gramsch and M. Rigol, Phys. Rev. A 86, 053615 (2012).

[30] J. Lancaster and A. Mitra, Phys. Rev. E 81, 061134 (2010).

[31] P. R. Zangara, A. D. Dente, E. J. Torres-Herrera, H. M. Pastawski, A. Iucci, and L. F. Santos, Phys. Rev. E 88, 032913 (2013).

[32] E. Lieb, T. Schultz, and D. Mattis, Ann. Phys. (N.Y.) 16, 407 (1961).

[33] P. Pfeuty, Ann. Phys. (N.Y.) 57, 79 (1970).

[34] P. Calabrese and J. Cardy, Phys. Rev. Lett. 96, 136801 (2006).

[35] S. Sachdev, Quantum Phase Transitions (Wiley Online Library, New York, 2007).

[36] E. Barouch, B. M. McCoy, and M. Dresden, Phys. Rev. A 2, 1075 (1970).

[37] F. Iglói and H. Rieger, Phys. Rev. Lett. 85, 3233 (2000).

[38] S. B. Rutkevich, Ukr. Phys. J. 25, 1135 (1980); translation available at arXiv:1201.0578.

[39] See Supplemental Material at http://link.aps.org/ supplemental/10.1103/PhysRevLett.118.130601 for a brief discussion on the splitting of the many-particle transition energies, the derivation of the many-particle dephasing prediction and its applicability, numerical results on relevant time scales, and a discussion of possible experimental realizations, which includes Refs. [40-45].

[40] J. Smith, A. Lee, P. Richerme, B. Neyenhuis, P. W. Hess, P. Hauke, M. Heyl, D. A. Huse, and C. Monroe, Nat. Phys. 12, 907 (2016).

[41] P. W. Hess (private communication).

[42] M. H. Devoret and R. J. Schoelkopf, Science 339, 1169 (2013).

[43] D. H. Slichter, C. Müller, R. Vijay, S. J. Weber, A. Blais, and I. Siddiqi, New J. Phys. 18, 053031 (2016).

[44] S. Hacohen-Gourgy, V. V. Ramasesh, C. D. Grandi, I. Siddiqi, and S. M. Girvin, Phys. Rev. Lett. 115, 240501 (2015).

[45] R. C. Bialczak, M. Ansmann, M. Hofheinz, M. Lenander, E. Lucero, M. Neeley, A. D. O’Connell, D. Sank, H. Wang, M. Weides, J. Wenner, T. Yamamoto, A. N. Cleland, and J. M. Martinis, Phys. Rev. Lett. 106, 060501 (2011). 